\title{
An apple (Malus domestica) AP2/ERF transcription factor modulates carotenoid accumulation
}

\author{
Qingyuan Dang ${ }^{1}$, Haiyun Sha', Jiyun Nie ${ }^{1}$, Yongzhang Wang ${ }^{1}$, Yongbing Yuan ${ }^{1 凶}$ and Dongjie Jia $\mathbb{B}^{1 凶}$
}

\begin{abstract}
Color is an important trait for horticultural crops. Carotenoids are one of the main pigments for coloration and have important implications for photosynthesis in plants and benefits for human health. Here, we identified an APETALA2 (AP2)/ETHYLENE RESPONSE FACTOR (ERF) transcription factor named MdAP2-34 in apple (Malus domestica Borkh.). MdAP2-34 expression exhibited a close correlation with carotenoid content in 'Benin Shogun' and 'Yanfu 3' fruit flesh. MdAP2-34 promotes carotenoid accumulation in MdAP2-34-OVX transgenic apple calli and fruits by participating in the carotenoid biosynthesis pathway. The major carotenoid contents of phytoene and $\beta$-carotene were much higher in overexpressing MdAP2-34 transgenic calli and fruit skin, yet the predominant compound of lutein showed no obvious difference, indicating that MdAP2-34 regulates phytoene and $\beta$-carotene accumulation but not lutein. MdPSY2-1 (phytoene synthase 2) is a major gene in the carotenoid biosynthesis pathway in apple fruit, and the MdPSY2-1 gene is directly bound and transcriptionally activated by MdAP2-34. In addition, overexpressing MdPSY2-1 in apple calli mainly increases phytoene and total carotenoid contents. Our findings will advance and extend our understanding of the complex molecular mechanisms of carotenoid biosynthesis in apple, and this research is valuable for accelerating the apple breeding process.
\end{abstract}

\section{Introduction}

Color is an important trait for horticultural crops, such as fruits, vegetables, and flowers ${ }^{1}$. Carotenoids are one of the main pigments for coloration, showing yellow, orange, red, or others in horticultural reproductive organs ${ }^{2}$. Carotenoids are important components for photosynthesis and are involved in light harvesting and photoprotection, plant growth, and development ${ }^{3}$. Carotenoids are valuable molecules that are beneficial for human health and animal survival, and carotenoids are also precursors of provitamin A and are antioxidants for reducing various chronic diseases ${ }^{4,5}$.

The biosynthetic pathway of carotenoids is well known in a variety of plant species, and it is modulated by many factors, such as limiting and metabolic enzyme steps, the

\footnotetext{
Correspondence: Yongbing Yuan (yyb@qau.edu.cn) or Dongjie Jia (jdj1985@126.com)

'Qingdao Key Lab of Modern Agriculture Quality and Safety Engineering, College of Horticulture, Qingdao Agricultural University, Qingdao 266109,
} China availability of storage organs and structures, or transcription factors (TFs) ${ }^{2,6,7}$. The structural genes encoding metabolic enzymes in the carotenoid pathway, including phytoene synthase (PSY), phytoene desaturase (PDS), $\zeta$ carotene isomerase (ZISO), zeta-carotene desaturase $(Z D S)$, carotenoid isomerase (CRTISO), lycopene $\beta$-cyclase $(L C Y-\beta)$, lycopene $\varepsilon$-cyclase $(L C Y-\varepsilon)$, beta-carotene hydroxylase $(C H Y-\beta)$, zeaxanthin epoxidase $(Z E P)$, carotenoid cleavage dioxygenases (CCD), and 9-cisepoxycarotenoid dioxygenase (NCED), have previously been characterized ${ }^{2}$. Previous studies have shown that PSYs play a significant role in carotenoid accumulation ${ }^{8,9}$. Transgenic tomato fruits and potato tubers overexpressing PSY show increased levels of total carotenoids $^{10,11}$. Multiple PSY genes modulate carotenoid accumulation in many species, such as maize, rice, loquat, and apple ${ }^{12-15}$. CaPSY2 can increase carotenoid content and compensate for the absence of PSY1 in pepper (Capsicum spp.) fruit ${ }^{16}$. OR/OR-like proteins influence carotenoid content by affecting PSY protein levels 
through posttranscriptional regulation in Arabidopsis and sweet potato ${ }^{17,18}$. For the other limiting genes, the downregulation of $L C Y$ - $\varepsilon$ increases the $\beta$-carotene content in potato ${ }^{19}$. The expression of the NCED gene results in a low $\beta$-carotenoid content in the white apricot cultivar $^{20}$. Inhibition of BoaCRTISO expression in Chinese kale affected carotenoid pathways and led to decreased carotenoid concentrations $^{21}$. The Orange gene $\mathrm{CmOr}$ regulates $\beta$-carotene accumulation in melon fruit by repressing $\beta$-carotene turnover and posttranslationally enhancing carotenogenesis in chromoplasts ${ }^{22}$.

A number of TFs have been identified to be involved in the carotenoid pathway. AGAMOUS-like 1 and FRUITFULL, which belong to MADS-box TFs, regulate carotenoid content in tomato ${ }^{23}$, and CsMADS6 modulates carotenoid metabolism by directly regulating $L C Y b 1$ in sweet orange $^{24}$. The NAC TFs SINAC1, SINAC4, and CpNAC1 regulate carotenoid accumulation during tomato and papaya fruit ripening ${ }^{25-27}$. MYB TFs, RCP1 in Mimulus lewisii flowers ${ }^{28}$, CrMYB68 in citrus ${ }^{29}$, and AdMYB7 in kiwifruit ${ }^{30}$, are all involved in carotenoid regulation. The R2R3-MYB TF WP1 together with MtTT8 and MtWD40-1 promotes floral carotenoid accumulation by directly activating the expression of MtLYCe and MtLYCb in Medicago petals ${ }^{31}$. CpbHLH1/2 regulates carotenoid biosynthesis and is related to carotenoid content during fruit ripening of papaya ${ }^{32}$. $S l \_$H2A. Z plays an essential role in the modulation of carotenoid content in tomato ${ }^{33}$.

The APETALA2 (AP2)/ETHYLENE RESPONSE FACTOR (ERF) superfamily contains four subfamilies according to the number of AP2/ERF domains and sequences, including AP2, CBF/DREB, ERF, and RAV ${ }^{34}$. AP2 subfamily proteins contain single or two AP2/ERF domains that are involved in the regulation of development $^{35}$. AP2/ERF proteins can bind to DNA cis-acting elements, including DRE (CCGAC), GCC box (AGCCGCC), (A/G)CC(G/C)AC, and AA(T)TTCAAA ${ }^{36}$. The TFs of the AP2/ERF superfamily play important roles in growth, development, and stress response processes in higher plants ${ }^{34-37}$. PpERF3 activates ABA biosynthesis by positively regulating $\mathrm{PpNCED} / 3$ transcription in peach $^{38}$. AP2/ERF TFs have been reported to modulate carotenoid accumulation. SIERF6 negatively regulates carotenoid accumulation in tomato ${ }^{39}$. In Arabidopsis, ERFs and RAP2.2 promote carotenoid biosynthesis by binding to the PSY promoter ${ }^{40}$. However, whether and how AP2/ERF TFs modulate carotenoid metabolism in apple fruit are currently unknown.

Apple (Malus domestica Borkh.), which has high nutrient value, is one of the most economical fruit crops worldwide. Fruit flavor and color are major apple traits that determine consumer preferences. Because of differences in carotenoid accumulation, the flesh color varies from white to yellow in different apple genotypes ${ }^{41}$. Previous studies indicated that MdPSY2 played a dominant role in carotenoid accumulation in apple fruits and was activated by AP2/ERF TFs ${ }^{15,41}$. There was a strong positive correlation between the expression of the TF AP2D26 and PSY2 in apple fruit ${ }^{15}$. In this study, we found that the total carotenoid contents of 'Benin Shogun' fruits were significantly higher than those of 'Yanfu 3' fruits during the developmental period at 150 and 170 days after full bloom (DAFB). We characterized an AP2 TF named MdAP2-34 and found that it induced increases in carotenoid accumulation. MdAP2-34 was able to promote carotenoid levels by enhancing MdPSY2-1 promoter activity. Moreover, overexpressing MdPSY2-1 in apple calli mainly increases phytoene and total carotenoid contents. Our findings will advance and extend our understanding of the complex molecular mechanisms of carotenoid biosynthesis in apple.

\section{Materials and methods Plant materials}

Apple fruits of 'Benin Shogun' and 'Yanfu 3' were collected at 120, 150, and 170 days after full bloom (DAFB). 'Benin Shogun' and 'Yanfu 3' are bud mutations of 'Red Fuji'. Every treatment included six fruits. Leaves of 'Benin Shogun' were collected in late spring and used for DNA extraction. Apple fruits of Granny Smith used for the transient transformation were collected at 150 DAFB. The fruits and leaves were stored at $-78^{\circ} \mathrm{C}$ until use.

Apple calli were induced from the 'Orin' apple flesh and were used for the transformation ${ }^{42}$. The calli were cultured and subcultured on MS medium (Murashige and Skoog basal salt mixture) supplemented with $1.0 \mathrm{mg} / \mathrm{L}$ 2,4-dichlorophenoxyacetic acid (2,4-D) and $1.0 \mathrm{mg} / \mathrm{L}$ 6-benzylaminopurine (6-BA).

\section{Subcellular localization}

The subcellular localization of MdAP2-34 was conducted as previously described ${ }^{42}$. The open reading frame (ORF) sequence of MdAP2-34 without the stop codon was cloned and inserted into the pRI101:GFP expression vector. The fusion construct 35S:MdAP2-34:GFP was transformed into $N$. benthamiana leaf epidermal cells. Fluorescence was detected by confocal microscopy after 2 -3 days of transfection. DAPI (4',6-Diamidino-2-phenylin-dole; Invitrogen) was used to stain the nucleus.

\section{Total carotenoids extraction and measurement}

The extraction and determination of total carotenoids were performed using a method as described previously ${ }^{43}$. Each sample was extracted with acetone-hexane (1:1, vol/ vol). The total carotenoid content of the extracts was determined using a UV-2550 UV-vis spectrophotometer. 


\section{Quantitative measurement of carotenoid components}

Carotenoid components of transformed apple fruit skin and apple calli were measured by MetWare Company (http://www.metware.cn/) ${ }^{33}$. Carotenoid compounds were detected using the AB Sciex QTRAP6500 LC-MS/MS platform. Three replicates of each assay were performed. Apple fruit skin and apple calli were freeze-dried and then homogenized and powdered in a mill of $50 \mathrm{mg}$ of dried powder. The dried powder was then extracted with a mixed solution of $n$-hexane:acetone:ethanol. The extracts were analyzed using an LC-APCI-MS/MS system (UHPLC, ExionLC ${ }^{\mathrm{TM}} \mathrm{AD}$; https://sciex.com.cn/; MS, Applied Biosystems 6500 Triple Quadrupole, https:// sciex.com.cn/). The analytical and detection conditions were as follows: HPLC: column, YMC C30 $(3 \mu \mathrm{m}, 2 \times$ $100 \mathrm{~mm})$; solvent system, methanol:acetonitrile $(3: 1, \mathrm{~V} / \mathrm{V})$ (0.01\% BHT, $0.1 \%$ formic acid):methyl tert-butyl ether $(0.01 \% \mathrm{BHT})$; gradient program, $100: 0 \mathrm{~V} / \mathrm{V}$ at $0-3 \mathrm{~min}$, $100: 0 \mathrm{~V} / \mathrm{V}$ at $3-6 \mathrm{~min}, 58: 42 \mathrm{~V} / \mathrm{V}$ at $6-8 \mathrm{~min}, 20: 80 \mathrm{~V} / \mathrm{V}$ at 8-9 $\mathrm{min}, 5: 95 \mathrm{~V} / \mathrm{V}$ at $9 \mathrm{~min}, 100: 0 \mathrm{~V} / \mathrm{V}$ at 9.1-11 $\mathrm{min}$; flow rate, $0.8 \mathrm{~mL} / \mathrm{min}$; temperature, $28^{\circ} \mathrm{C}$; injection volume, $2 \mu \mathrm{L}$. The $\alpha$-carotene, antheraxanthin, violaxanthin, neoxanthin, phytofluene, phytoene, $\beta$-cryptoxanthin, and rubixanthin standards were purchased from BOC Chemicals. The zeaxanthin standard was purchased from Bide Chemicals. The $\beta$-carotene standard was purchased from RHAWN Chemicals. The Lutein standard was purchased from Aladdin Chemicals.

\section{RNA extraction, qRT-PCR assay, and extraction of genomic DNA}

Total RNA was isolated from fruit flesh of 'Benin Shogun' and 'Yanfu 3', fruit skin of transiently transformed 'Granny Smith' and transgenic apple calli (TAC), and qRT-PCR (reverse transcription-quantitative PCR) was carried out as previously described ${ }^{44}$. EF1 $\alpha$ was used to serve as the reference gene. The qRT-PCR assays were conducted with three biological replicates, and each biological replicate was conducted with three technical replicates. Genomic DNA was isolated form 'Granny Smith' leaves. All primers are used in Supplemental Table S1.

\section{Construction of plasmids and genetic transformation}

The overexpression and interference plasmids were constructed as previously described ${ }^{45}$. Each ORF sequence of MdAP2-34 and MdPSY2-1 was cloned and inserted into pRI101-FLAG (overexpression vector). A $728 \mathrm{bp}$ fragment (located between +5 and $+732 \mathrm{bp}$ of the ORF) of MdAP2-34 was cloned and inserted into pFGC5941 (RNAi vector). Every recombinant plasmid of 35S:MdAP2-34, RNAi:MdAP2-34, and 35 S:MdPSY2-1 was then transformed into Granny Smith apple fruit skin and Orin apple calli ${ }^{45}$. All primers are used in Supplemental Table S1.

\section{Proteins extraction and western blotting}

Protein extraction and western blotting assays were performed as previously described ${ }^{42,45}$. Anti-ACTIN antibody and anti-FLAG antibody were used, which were obtained from Beyotime Biotech Company.

\section{Yeast one-hybrid assay}

$\mathrm{Y} 1 \mathrm{H}$ (yeast one-hybrid) assays were conducted as previously described $^{45}$. The ORF sequence of $M d A P 2-34$ was cloned and inserted into the vector pJG4-5 (the effector vector) (Clontech, USA). Each promoter fragment (upstream of the ATG codon) of MdPSY1 (1725 bp), MdPSY2-1 (1733 bp), MdPSY2-2 (1850 bp), MdPDS (1659 bp), $M d Z D S 1$ (1800 bp), MdZDS2 (1683bp), MdLCY- $\beta$ (1748 bp), MdCHY- $\beta 2-1$ (1631 bp), MdZEP (1758 bp), and MdCCD1 (1782 bp) was cloned into the vector pLacZi (the reporter vector). All primers are used in Supplemental Table S1.

\section{EMSAs}

The EMSA was conducted as previously described ${ }^{45}$. The ORF sequence of MdAP2-34 was cloned into the vector pGEX-4T-1 (with GST-tag) and then transformed into BL21 (DE3) Escherichia coli cells for MdAP2-34 protein expression. The $5^{\prime}$ biotin end-labeled probes were synthesized by Sangon Biotech. The EMSA was performed using the LightShift ${ }^{\mathrm{TM}}$ Chemiluminescent EMSA Kit (Thermo Fisher Scientific, USA). The primers were used, and the biotin-labeled sequences of the MdPSY2-1 promoter are shown in Supplemental Table S1.

\section{ChIP-PCR assay}

The ChIP-PCR assay was performed following Jia et al. $^{45}$. The ORF of MdAP2-34 was inserted into the pRI101-FLAG vector (with $3 \times$ FLAG), and the fusion MdAP2-34-FLAG was generated. Recombinant pRI101FLAG-MdAP2-34 was transformed into apple calli, and the MdAP2-34-FLAG protein was extracted from transgenic calli. ChIP-PCR analysis was conducted using the EpiQuik $^{\mathrm{TM}}$ Plant ChIP Kit (Epigentek, USA) and antiDDDDK tag (Binds to FLAG® tag sequence) antibody (ab125243, Abcam, UK). Three promoter regions of MdPSY2-1 were used to assess their enrichment. Every ChIP assay was conducted three replicates. The primers used are in Supplemental Table S1.

\section{LUC assay}

The LUC analysis was conducted as previously described $^{45}$. The ORF of MdAP2-34 was inserted into pGreenII $62-S K$ (the effector vector) under the control of the CaMV35S promoter. The promoter fragment of MdPSY21 was inserted into pGreenII 0800-LUC (the reporter vector). The vectors of the effector and reporter were cotransformed into 4-week-old $N$. benthamiana leaves. 
The luciferase signals of Firefly and Renilla were observed and measured using an Infinite M200 (Tecan, Switzerland). Every LUC analysis was conducted six replicates. The primers used are in Supplemental Table S1.

\section{Statistical analysis}

In the experimental design used, the data were tested and analyzed for three biological replicates, and each biological replicate was analyzed three technical repetitions. Significant differences were determined using GraphPad Prism 5 software ${ }^{42,45}$ (* represents $P<0.05$; *** represents $P<0.01$; *** represents $P<0.001$ ).

\section{Results}

Characterization of MdAP2-34 associated with carotenoid content in 'Benin Shogun' and 'Yanfu 3' apple fruits

A previous study indicated that the AP2 subfamily consists of 51 genes in apple, which contain single or double AP2 domain ${ }^{34}$. Based on the annotation of the website (http://bioinformatics.cau.edu.cn/AppleMDO/) ${ }^{46}$, 12 AP2 TFs were identified that could be expressed in apple fruits (Supplemental Table S2). In the 'Benin Shogun' and 'Yanfu 3' fruit flesh (Fig. 1a), the total carotenoid content increased during the developmental period at 120, 150, and 170 DAFB (Fig. 1b). The total carotenoid content of 'Benin Shogun' fruits was significantly higher than that of 'Yanfu 3' fruits at 150 and 170 DAFB (Fig. 1b). Pearson's correlation $(r)$ between each AP2 TF transcript levels and total carotenoid content in apple flesh of 'Benin Shogun' and 'Yanfu 3' indicated that the expression level of MdAP2-34 was significantly positively associated with carotenoid contents (Supplemental Table S2). However, the MdAP2-24 expression level was inconsistent with the total carotenoid content in 'Yanfu 3' fruit flesh, and the MdAP2-44 expression level was negatively correlated with the total carotenoid content, so the two genes were filtered out. The transcript level of $M d A P 2-34$ increased in both 'Benin Shogun' and 'Yanfu 3' fruit flesh during the developmental period at 120, 150, and 170 DAFB (Fig. 1c). The expression level of MdAP2-34 in 'Benin Shogun' fruits was significantly higher than that in 'Yanfu 3' fruits at 150 and 170 DAFB (Fig. 1c). Furthermore, the expression of MdAP2-34 showed a close correlation ( $r=$ 0.9844, $P<0.001$ ) with carotenoid content in 'Benin Shogun' and 'Yanfu 3' fruit flesh (Fig. 1d). Therefore, MdAP2-34 was selected as a candidate gene associated with carotenoid accumulation.

MdAP2-34 (MD17G1226700 or MDP0000231748) is a typical AP2/ERF TF. MdAP2-34 contains an ORF of $1,101 \mathrm{bp}$ and encodes a predicted 367 amino acid (aa) protein. MdAP2-34 was predicted by NCBI (https://www. ncbi.nlm.nih.gov/Structure/cdd) to contain two conserved AP2 domains (49-121 aa, 151-13 aa) (Supplemental Fig. S1a). Phylogenetic analysis showed that MdAP2-34 and
AtAIL5 have high homology (Supplemental Fig. S1b), and previous results indicated that AtAIL5 could induce a larger floral organ phenotype ${ }^{47}$. To investigate the subcellular localization of the MdAP2-34 protein, the ORF of MdAP2-34 was fused to EGFP (MdAP2-34:GFP) and transiently expressed in Nicotiana benthamiana leaves. The vector pRI101-GFP (35S:GFP) was used as a control. The results indicated that fusion protein signals were identified in the nucleus (Fig. 1e).

\section{MdAP2-34 positively regulates carotenoid accumulation in transgenic lines}

To confirm whether MdAP2-34 could modulate carotenoid accumulation, the constructs of 35S:MdAP2-34 and RNAi:MdAP2-34 (Fig. 2a) were transformed into apple calli, and the empty vectors of pRI101-flag (P101F) and pFGC5941 (P5941) served as controls (Fig. 2b). The MdAP2-34-FLAG protein was extracted and detected in MdAP2-34-overexpressing transgenic calli (Supplemental Fig. S2). The MdAP2-34 expression level was remarkably higher in MdAP2-34-OVX transgenic calli than in P101F calli but much lower in the MdAP2-34-RNAi calli than in P5941 calli (Fig. 2c). The total carotenoid content was significantly higher in MdAP2-34-OVX calli $(22.33 \mu \mathrm{g} / \mathrm{g}$ FW) than in P101F calli (11.87 $\mu \mathrm{g} / \mathrm{g}$ FW), with an increase of $88.12 \%$ (Fig. 2d; Table 1). The total carotenoid content was lower in MdAP2-34-RNAi calli $(6.33 \mu \mathrm{g} / \mathrm{g} \mathrm{FW})$ than in P5941 calli $(9.25 \mu \mathrm{g} / \mathrm{g} \mathrm{FW})$, with a reduction of $31.57 \%$ (Fig. 2d; Table 1). For the carotenoid compounds, in total, 12 carotenoids were isolated from TAC (Table 1). Among these carotenoids, $\beta$-carotene, $\beta$-cryptoxanthin, phytoene, and violaxanthin were the major carotenoids present (Table 1). The contents of four specific carotenoids ( $\beta$-carotene, $\beta$-cryptoxanthin, phytoene, and violaxanthin) were remarkably higher in MdAP2-34-OVX calli than in P101F calli and were much lower in MdAP2-34-RNAi calli than in P5941 calli (Table 1). These results suggest that MdAP2-34 enhances the carotenoid contents in apple calli.

A transient expression assay based on Agrobacterium tumefaciens infiltration was used to overexpress or silence MdAP2-34 in the fruit skin of Granny Smith (Fig. 2e). After 14 days of the transient transformation of Granny Smith, the MdAP2-34-OVX Granny Smith fruit skin was harvested (Fig. 2e). The expression level of MdAP2-34 was remarkably higher in MdAP2-34-OVX fruit skin than in P101F fruit skin but much lower in MdAP2-34-RNAi fruit skin than in P5941 fruit skin (Fig. 2f). The total carotenoid content was significantly higher in MdAP2-34-OVX fruit skin $(24.33 \mu \mathrm{g} / \mathrm{g} F W)$ than in P101F fruit skin $(15.15 \mu \mathrm{g} / \mathrm{g}$ FW), with an increase of $60.59 \%$ (Fig. 2g; Table 1). The total carotenoid content was lower in MdAP2-34-RNAi fruit skin $(12.33 \mu \mathrm{g} / \mathrm{g}$ FW) than in P5941 fruit skin $(17.25 \mu \mathrm{g} / \mathrm{g}$ FW), with a reduction of $28.52 \%$ (Fig. $2 \mathrm{~g}$; 

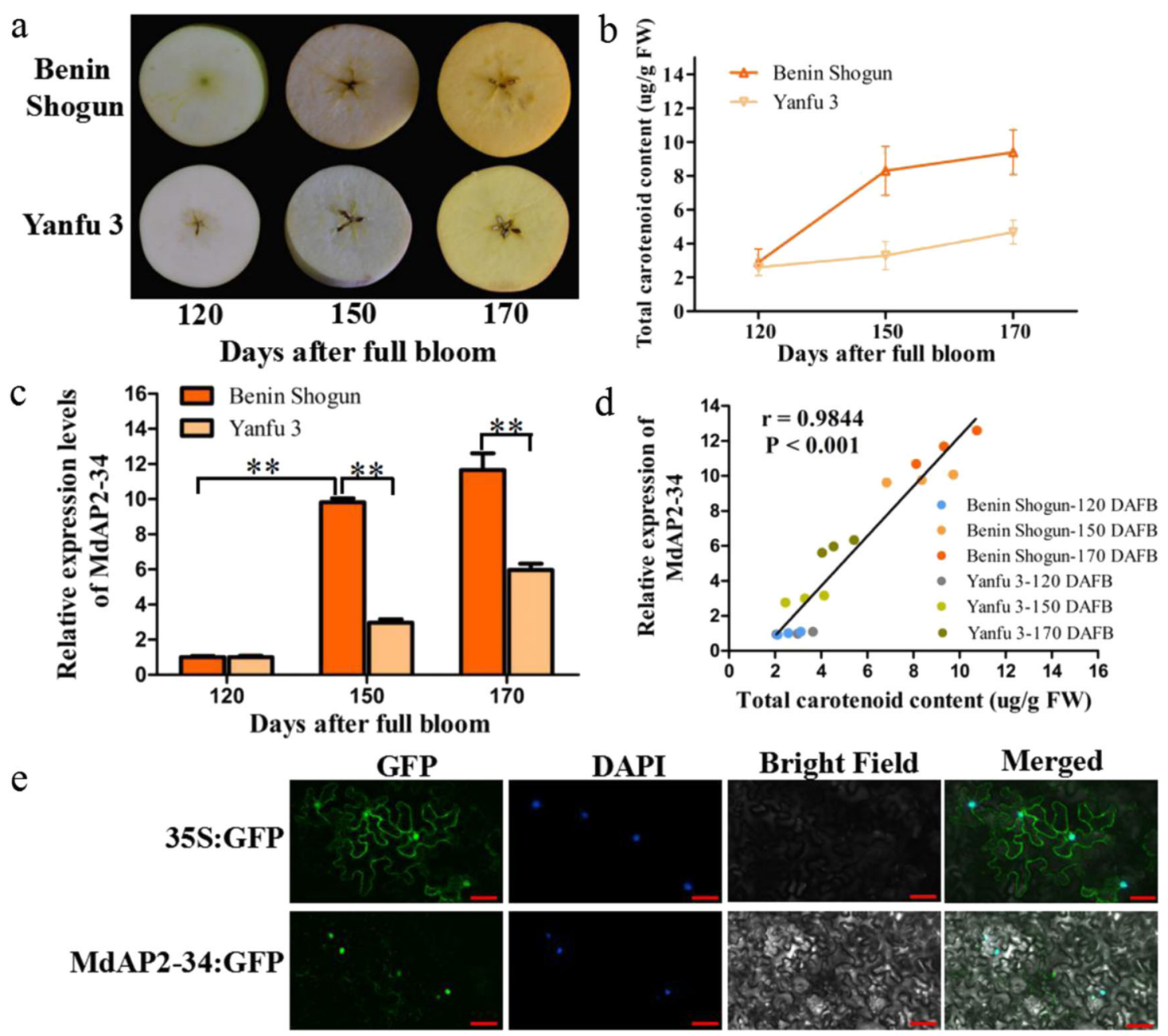

Fig. 1 MdAP2-34 was identified as a candidate gene associated with carotenoid content. a Phenotypes of 'Benin Shogun' and 'Yanfu 3' fruits at 120, 150, and 170 DAFB. b The total carotenoid content in 'Benin Shogun' and 'Yanfu 3' fruit flesh at 120, 150, and 170 DAFB. c The transcript level of MdAP2-34 in 'Benin Shogun' and 'Yanfu 3' fruit flesh at 120, 150, and 170 DAFB. d Correlation analysis between the expression of MdAP2-34 and fruit carotenoid content in 'Benin Shogun' and 'Yanfu 3' fruit flesh. e Subcellular localization of transiently expressed MdAP2-34:GFP fusion protein and the control 35S:GFP in Nicotiana benthamiana leaves. 6-Diamidino-2-phenylindole (DAPI) was used to stain the nucleus. Fluorescence images were observed and obtained using confocal microscopy. Scale bars $=50 \mu \mathrm{m}$. Values are the mean \pm SE in $(\mathbf{b}, \mathbf{c})$

Table 1). For the carotenoid compounds, in total, 19 carotenoids were isolated from transgenic Granny Smith fruit skin (Table 1). Among these carotenoids, $\beta$-carotene, phytoene, and lutein were the major carotenoids present. The ratios of $\beta$-carotene, phytoene, and lutein to total carotenoids in P101F 'Granny Smith' fruit skin were 16.63, 13.86 , and $58.42 \%$, respectively (Table 1 ). The contents of four specific carotenoids ( $\beta$-carotene, $\beta$-cryptoxanthin, phytoene, and violaxanthin) were remarkably higher in MdAP2-34-OVX fruit skin than in P101F fruit skin and were remarkably lower in MdAP2-34-RNAi calli than in P5941 calli (Table 1). These findings suggest that MdAP234 plays an important role in the carotenoid accumulation and positively regulates carotenoid accumulation in the fruit skin of apple trees.
Expression of carotenoid biosynthesis genes in transgenic fruit skin and calli

The structural genes encoding enzymes of carotenoid biosynthesis include PSY, PDS, ZISO, ZDS, CRTISO, LCY- $\beta$, $C H Y-\beta, \quad Z E P, \quad V D E, \quad C C S, \quad C C D$, and NCED (Supplemental Fig. S3), which have previously been characterized $^{2}$. The expression levels of genes related to carotenoid biosynthesis were measured and analyzed in TAC and transgenic Granny Smith fruit skin (TFS) using qRT-PCR (Fig. 3). The expression levels of MdPSY1, MdPSY2-1, MdPDS, MdZDS1, MdZDS2, MdLCY- $\beta$, $M d C H Y-\beta 2-1$, and $M d C C D 1$ were significantly higher in MdAP2-34-OVX TAC than in P101F TAC and were remarkably lower in MdAP2-34-RNAi TAC than in P5941 TAC. The expression levels of MdPSY2-1, MdPDS, MdZ- 


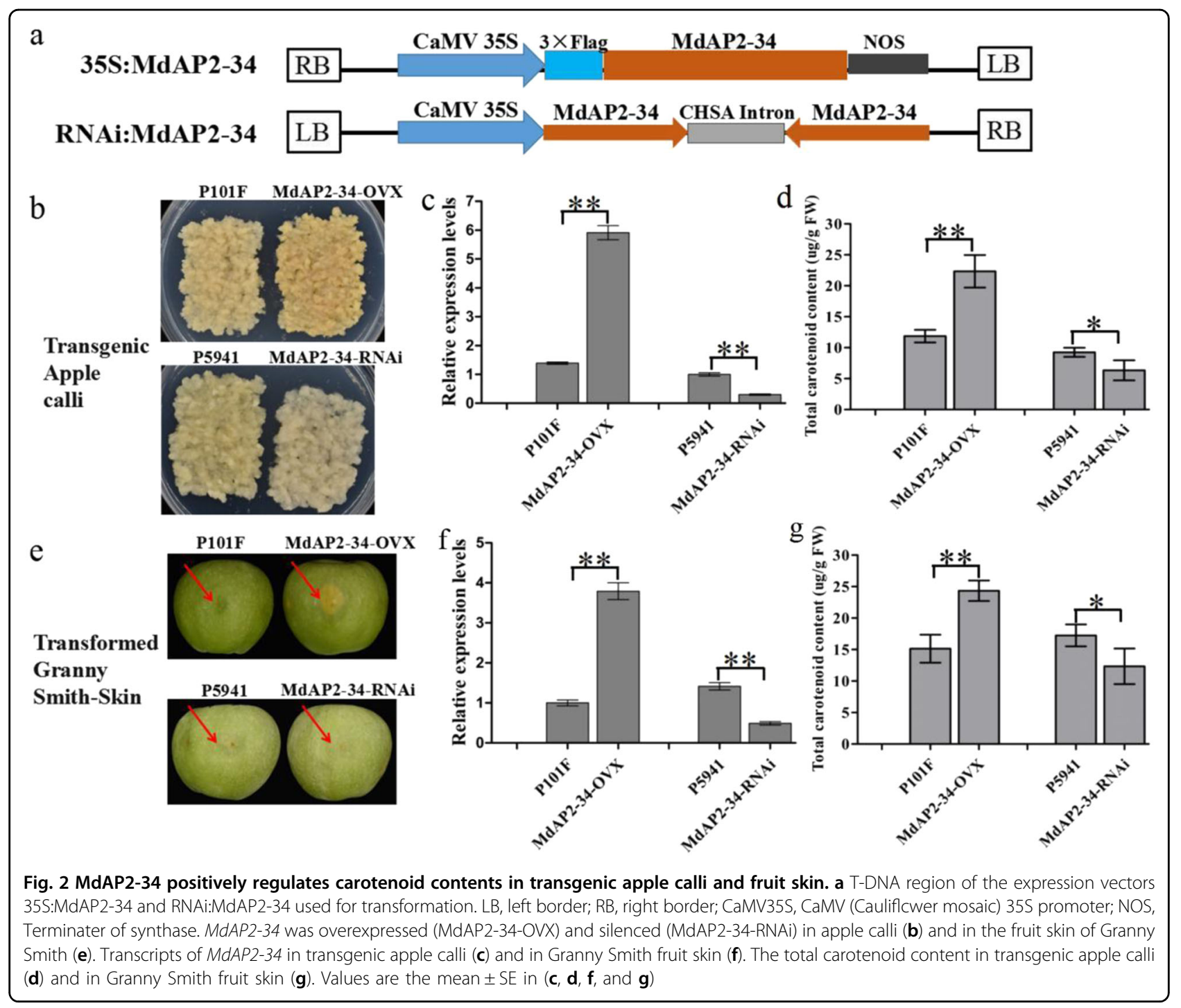

ISO, MdZDS1, MdCRTISO, MdLCY- $\beta, \quad M d C H Y-\beta 2-1$, $M d Z E P, M d C C S 1$, and $M d C C D 4$ were significantly higher in the MdAP2-34-OVX TFS than in the P101F TFS and were remarkably lower in the MdAP2-34-RNAi TFS than in the P5941 TFS. The expression levels of MdPSY2-1, MdPDS, $M d Z D S 1, M d L C Y-\beta$, and $M d C H Y-\beta 2-1$ were markedly upregulated in both TAC and TFS of MdAP2-34-OVX compared to P101F, which were markedly downregulated in both TAC and TFS of MdAP2-34-RNAi compared to P5941 (Fig. 3). These results correspond to the detected differences in carotenoid levels between MdAP2-34-OVX and P101F, and between MdAP2-34-RNAi and P5941 in TAC and TFS, indicating that carotenoid levels are influenced by most carotenoid biosynthetic genes in the carotenoid biosynthesis pathway and a positive correlation between MdAP2-34 expression levels and those of the genes MdPSY2-1, MdPDS, $M d Z D S 1, M d L C Y-\beta$, and $M d C H Y-\beta 2-1$.

\section{MdAP2-34 enhances the transcription of MdPSY2-1 by} directly binding to its promoter

To test how MdAP2-34 modulates carotenoid accumulation, a yeast one-hybrid $(\mathrm{Y} 1 \mathrm{H})$ analysis was conducted to detect whether MdAP2-34 directly regulates the transcription of MdPSY1, MdPSY2-1, MdPSY2-2, MdPDS, $M d Z D S 1, M d Z D S 2, M d L C Y-\beta, M d C H Y-\beta 2-1, M d Z E P$, and $M d C C D 1$. We found that MdAP2-34 was able to directly bind to the MdPSY2-1 promoter (Fig. 4a). An EMSA was conducted to further confirm the binding interaction. Previous studies reported that many DREB proteins could specifically bind to a core sequence (A/G) CCGAC of the dehydration-responsive element (DRE), such as AtDREB1A and AtDREB2A ${ }^{48,49}$. Here, we found an ACCGAC motif in the MdPSY2-1 promoter (Fig. 4b). MdAP2-34 was able to bind to the ACCGAC motif in the MdPSY2-1 promoter (Fig. 4b). 


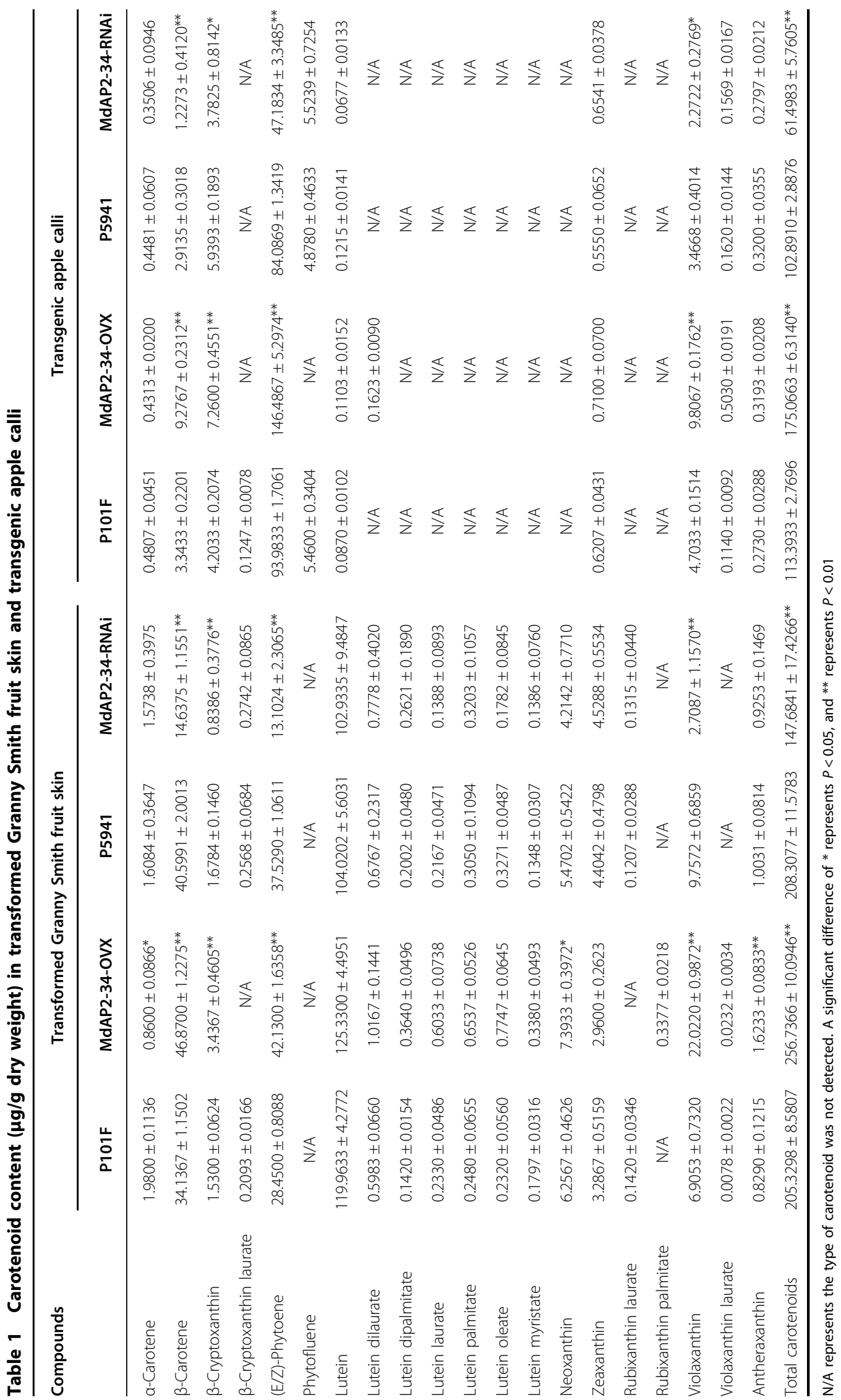






To confirm the binding of MdAP2-34 to the MdPSY2-1 promoter in vivo, a ChIP-PCR (chromatin immunoprecipitation-PCR) assay was performed. The MdAP2-34FLAG fusion protein was overexpressed and synthesized in TAC, and P101F was used as a control. The presence of MdAP2-34 enhanced the PCR-based detection of the MdPSY2-1 promoter (Fig. 4c), indicating that MdAP2-34 can also bind to the MdPSY2-1 promoter in vivo. Furthermore, to confirm how MdAP2-34 may affect the activity of the MdPSY2-1 promoter, a luciferase (LUC) transactivation assay in leaves of wild tobacco (Nicotiana benthamiana) through cotransformation with Pro35S: MdAP2-34 and ProMdPSY2-1:LUC constructs was conducted. Three combinations of ProMdPSY2-1:LUC
(ProMdPSY2-1) and Pro35S:MdAP2-34 (MdAP2-34) were used, including ProMdPSY2-1 to MdAP2-34 ratios of 1:1, 1:5, and 1:9, respectively. When MdAP2-34 was cotransformed with ProMdPSY2-1, MdPSY2-1 promoter activity was significantly increased. As the ProMdPSY2-1 to MdAP2-34 ratio increased from 1:1 to 1:9, the promoter activity of MdPSY2-1 was significantly increased (Fig. 4d). Taken together, these results support MdAP234 activation of MdPSY2-1 transcription.

\section{Overexpressing MdPSY2-1 in apple calli increases carotenoid content}

The 35S:MdPSY2-1 construct (Fig. 5a) was transformed into apple calli, and the empty vector P101F was used as a 


\section{a MdAP2-34 + Placzi \\ MdAP2-34 + MdPSY2-1 \\ pJG4-5 + MdPSY2-1 \\ MdAP2-34 + MdPSY1 \\ MdAP2-34 + MdPSY2-2 \\ MdAP2-34 + MdPDS \\ MdAP2-34 + MdZDS1 \\ MdAP2-34 + MdZDS2 \\ MdAP2-34 + MdLCY- $\beta$ \\ MdAP2-34 + MdCHY-B2-1 \\ MdAP2-34 + MdZEP \\ MdAP2-34 + MdCCD1}

\section{$\mathrm{SD} / \mathrm{-T} / \mathrm{-U}$}

$\mathrm{SD} /-\mathrm{T} /-\mathrm{U}+$

C

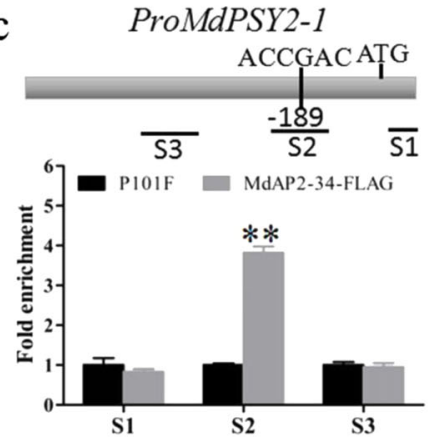

d

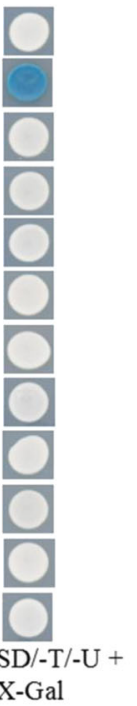

b

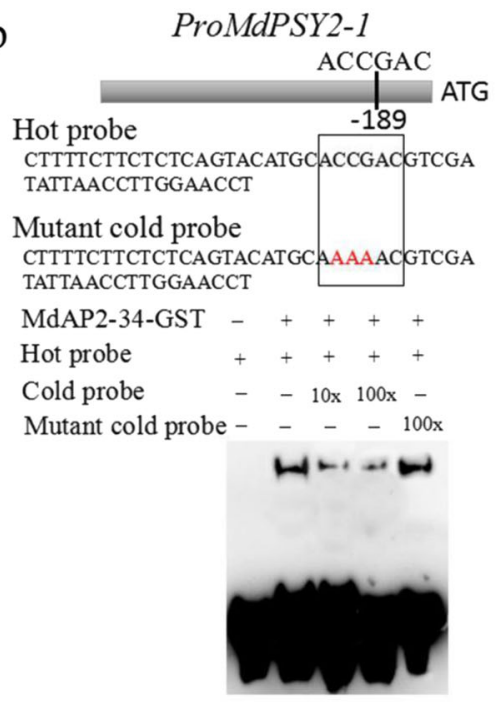

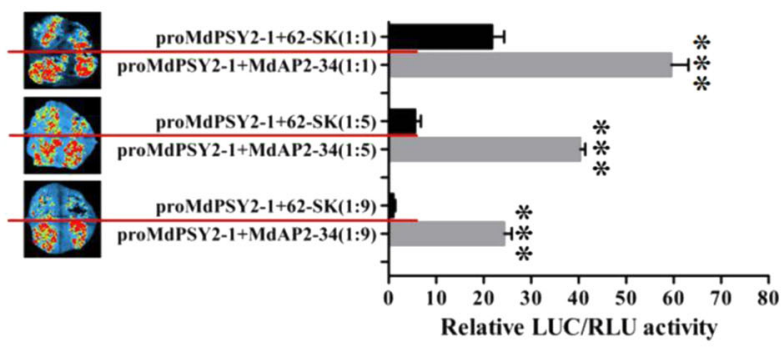

Fig. 4 MdAP2-34 enhances the MdPSY2-1 transcription. a Y1H assay showing that MdAP2-34 binds to the MdPSY2-1 promoter. $\mathbf{b}$ EMSA showing that MdAP2-34 binds to the ACCGAC motif of the MdPSY2-1 promoter. c ChIP-PCR assay showing that MdAP2-34 binds to the MdPSY2-1 promoter in vivo. Three regions (S1-S3) in MdPSY2-1 were explored. $\mathbf{d}$ LUC activity analysis showing that MdAP2-34 enhances the MdPSY2-1 promoter. Three concentrations of the combination of ProMdPSY2-1:LUC (ProMdPSY2-1) and Pro35S:MdAP2-34 (MdAP2-34) were set up, for which the ratio of ProMdPSY2-1 to MdAP2-34 was 1:1, 1:5, and 1:9. Values are the mean \pm SE in $(\mathbf{c}, \mathbf{d})$

control (Fig. 5b). The MdPSY2-1 expression level was significantly higher in overexpressing transgenic calli (MdPSY2-1-OVX) than in P101F calli (Fig. 5c). The total carotenoid, phytoene, phytofluene, and $\beta$-carotene contents were markedly higher in MdPSY2-1-OVX calli than in P101F calli (Fig. 5d). These results indicate that overexpressing MdPSY2-1 in apple calli mainly increases phytoene, phytofluene, $\beta$-carotene, and total carotenoid contents and that MdPSY2-1 plays an important role in carotenoid accumulation.

\section{Discussion}

Carotenoids are important pigments for coloration and nutrition in horticultural plants and play important roles in plant growth and development. Apples with healthful metabolites and high nutritional value are consumed worldwide. Recently, carotenoid content and components have become important breeding objectives in apple ${ }^{30,41}$. In this study, we characterized the AP2-type TF MdAP2-
34 in Benin Shogun apple, in which ripened fruits appeared orange. Through experiments, we developed a model that explains the molecular basis for MdAP2-34. We found that MdAP2-34 enhances carotenoid accumulation. MdAP2-34 was able to promote carotenoid levels by activating MdPSY2-1 promoter activity (Fig. 6).

Apples are consumed in large quantities worldwide because of their healthy metabolites. Carotenoid pigments contribute to the skin coloration of apple fruit; however, their concentrations are very low in the apple flesh. A previous study suggested that they have low concentrations of carotenoids in the fruit of commercial apple cultivars $(<2.5 \mu \mathrm{g} / \mathrm{g}$ fresh weight) but high fruit carotenoid concentrations in the rootstock cultivar 'Aotea ${ }^{41}$. The study indicated that the concentration of total carotenoids in the fruit of the commercial cultivar 'Benin Shogun' (with flesh color of orange, Fig. 1a) was up to $9.4 \mu \mathrm{g} / \mathrm{g}$ fresh weight at 170 DAFB, which was much higher than that of 'Yanfu 3' and the carotenoid concentrations reported previously. The results suggest that 


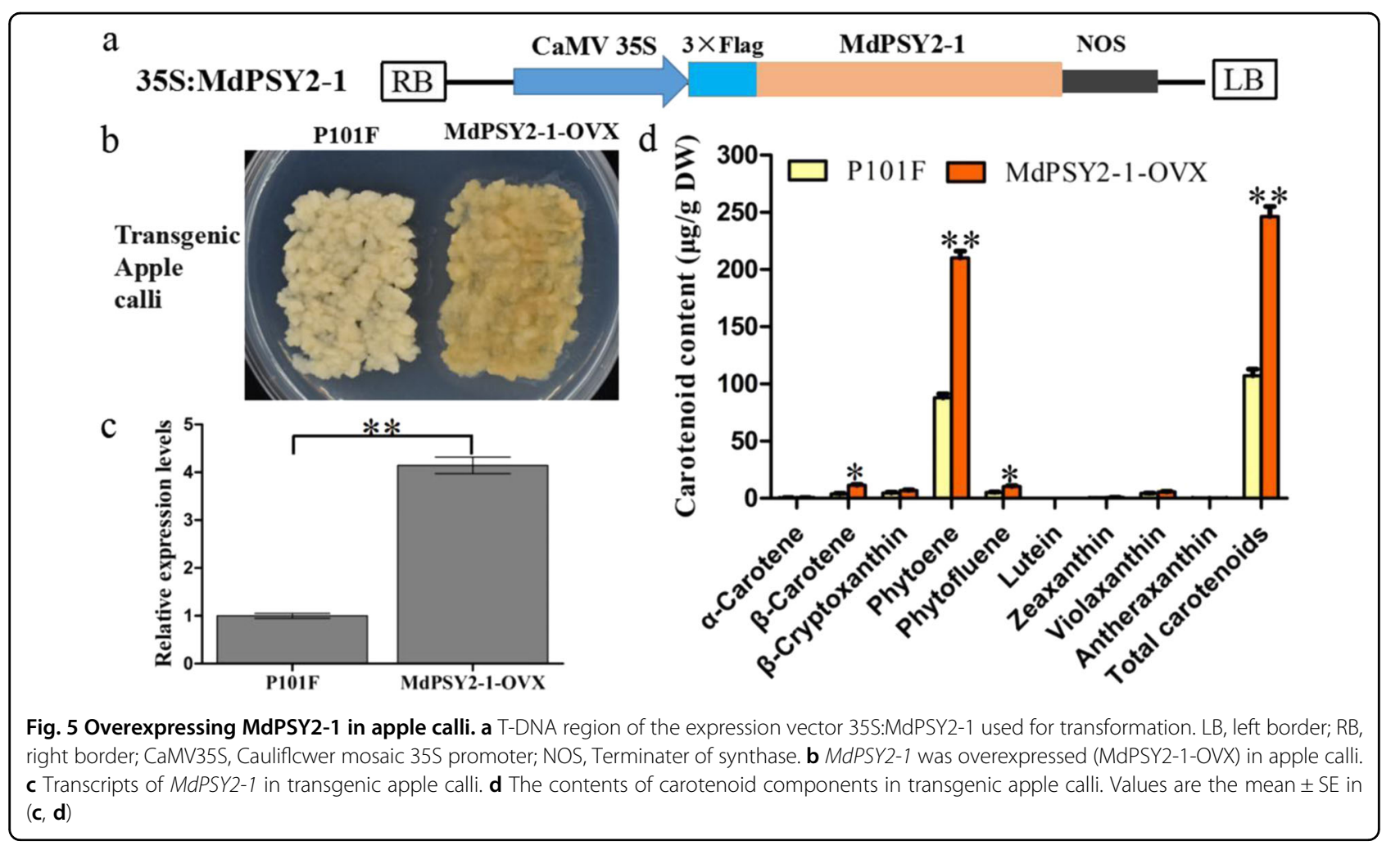

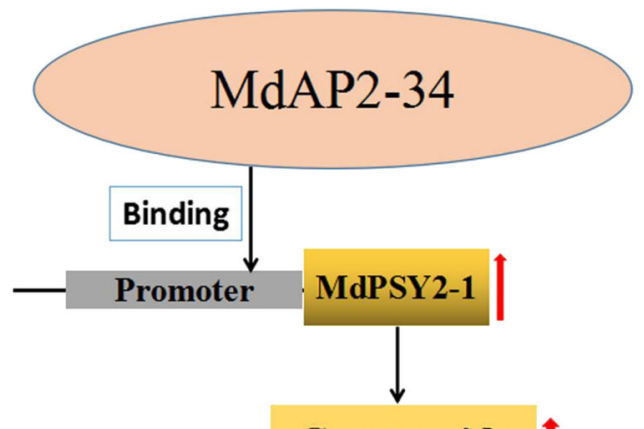

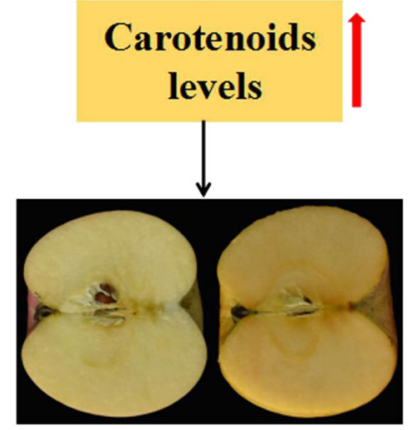

Fig. 6 Proposed model for the role of MdAP2-34 in regulating carotenoid accumulation in apple. MdAP2-34 modulates carotenoid accumulation in apple by participating in the carotenoid biosynthesis pathway. MdAP2-34 could modulate carotenoid accumulation by directly binding to the MdPSY2-1 promoter and enhancing MdPSY2-1 transcriptional activity the commercial cultivar 'Benin Shogun' may be better for research on the carotenoid metabolic mechanism. Our future study is intended to focus on the mechanisms of carotenoid biosynthesis in different apple cultivars, including the full transcriptome, genetics, and epigenetics. The cause of different genetic variations in carotenoid accumulation in Malus will help us to understand the mechanisms of carotenoid accumulation in apple and will be beneficial for the breeding of new apple cultivars with high carotenoid concentrations.

Many TFs play important roles in carotenoid biosynthesis. It is well known that AP2/ERFs participate in many plant development processes and play important roles in regulating fruit quality and pigment biosynthesis $^{37,50}$. However, AP2/ERF TFs have rarely been reported to regulate carotenoid biosynthesis in apple fruits. In this study, based on the previous analysis of the AP2/ERF family $^{34}$ and the annotation of websites (http:// bioinformatics.cau.edu.cn/AppleMDO/ $/)^{46}$, we identified 12 AP2 TFs from the AP2 subfamily expressed in apple fruits. By correlation analysis, we characterized MdAP234 as a candidate AP2 TF associated with carotenoid accumulation (Supplemental Table S2). The study only identified the AP2 subfamily, and other AP2/ERF subfamilies may also modulate carotenoid accumulation in apple fruit and requires further research. MdAP2-34 contains two conserved AP2 domains (Fig. 1e); however, the other reported carotenoid-related AP2/ERF TFs 
contain only one AP2 domain (Supplementary Fig. S1a). MdAP2-34 is a member of the AP2 subfamily, which has high homology with AtAIL5 in Arabidopsis, and AtAIL5 could induce a larger floral organ phenotype ${ }^{47}$. MdAP234 may have a similar function, and further studies are required to test its function.

It has been reported that AP2/ERF superfamily TFs can modulate carotenoid biosynthesis. With regard to carotenoid regulation, RAP2.2 promotes carotenoid accumulation by binding to the PSY promoter in Arabidopsis ${ }^{40}$. However, SIERF6 represses carotenoid accumulation in tomato, and reduced SIERF6 expression results in increased carotenoid concentration $^{39}$. This study indicated that overexpressing MdAP2-34 in transgenic calli and fruits enhanced carotenoid accumulation (Fig. 2 and Table 1). The contents of four specific carotenoids ( $\beta$-carotene, $\beta$-cryptoxanthin, phytoene, and violaxanthin) were significantly higher in MdAP2-34OVX fruit skin of transgenic Granny Smith than in P101F fruit skin and were remarkably lower in MdAP2-34-RNAi calli than in P5941 calli (Table 1). The predominant carotenoid compounds differed among different apple culti$\operatorname{vars}^{41}$. In ripening apple fruit flesh, the predominant compounds in 'Royal Gala' were violaxanthin and neoxanthin, those in 'Aotea' flesh were $\beta$-cryptoxanthin and $\beta$-carotene, and those in 'Granny Smith' were lutein. The study also indicated that lutein was the predominant compound in Granny Smith fruit skin, followed closely by $\beta$-carotene and phytoene (Table 1). The $\beta$-carotene and phytoene contents were much higher in MdAP2-34overexpressing transgenic calli and fruit skin, yet lutein showed no obvious difference, indicating that MdAP2-34 regulates $\beta$-carotene and phytoene accumulation but not lutein. MdAP2-34 could not modulate lutein-related genes, and other regulators or proteins may contribute to lutein accumulation in apple, which needs further study.

The structural genes encoding enzymes of carotenoid biosynthesis included MdPSY2-1, MdPDS, MdZDS1, $M d L C Y-\beta$, and $M d C H Y-\beta 2-1$, which were markedly upregulated in both MdAP2-34-OVX TAC and fruits and were markedly downregulated in both MdAP2-34-RNAi TAC and fruits (Fig. 3). MdAP2-34 could enhance carotenoid accumulation by activating MdPSY2-1 promoter activity. However, MdAP2-34 only directly binds to the MdPSY2-1 promoter but not the other carotenoid-related structural genes (Fig. 4a). There may be other mechanisms by which MdAP2-34 interacts with other TFs or genes to regulate carotenoid biosynthesis. Taken together, carotenoid accumulation in apple fruit is probably controlled by a complex regulatory network and warrants further study.

The genes encoding enzymes of carotenoid biosynthesis include PSY, PDS, ZISO, ZDS, CRTISO, LCY- $\beta$, CHY- $\beta$, $Z E P, V D E, C C S, C C D$, and NCED, which have previously been characterized ${ }^{2}$. Multiple $P S Y$ genes deserve functional diversity, some of which play important roles in carotenoid accumulation ${ }^{8,9}$. Previous studies indicated that PSYs modulate carotenoid accumulation in many fruits of plant species, such as tomato ${ }^{8}$, potato ${ }^{11}$, loquat $^{14}$, apple $^{15}$, and peppers ${ }^{16} . M d P S Y 2$ exhibited higher transcript levels than MdPSY1 in diverse apple cultivars, indicating that MdPSY2 could be mainly responsible for the first carotenoid pathway step and plays a dominant role in carotenoid accumulation in apple fruits ${ }^{15,41}$. The different expression levels of MdPSY1 and MdPSY2 in apple were influenced by their promoters and activated by AP2/ERF TFs. There was a strong positive correlation between the expression of the TF AP2D26 and PSY2 in apple fruit, revealing a potential regulatory relationship ${ }^{15}$. The role of PSYs in the carotenoid pathway was prominent in Arabidopsis, and the PSY promoter was implicated and activated by AtRAP2. ${ }^{40}$. The results indicated that MdPSY2-1 increases carotenoid content and was directly activated by MdAP2-34 (Figs. 4, 5). In addition, posttranscriptional regulation of PSY in controlling carotenoid biosynthesis has been reported, and PSYs are verified as the rate-limiting step in carotenogenesis ${ }^{17}$. In this study, by overexpressing the $M d P S Y 2-1$ gene in apple calli, the total carotenoid and phytoene contents were markedly increased (Fig. 5). The results show that MdPSY2-1 is a major gene in the carotenoid biosynthesis pathway in apple fruit.

Herein, we found that MdAP2-34 promotes carotenoid accumulation by participating in the carotenoid biosynthesis pathway. MdPSY2-1 is a major gene in the carotenoid biosynthesis pathway in apple fruit, and the MdPSY2-1 gene is directly bound and transcriptionally activated by MdAP2-34. Furthermore, overexpressing MdPSY2-1 in apple calli mainly increases phytoene and total carotenoid contents. This research is valuable for accelerating the apple breeding process and for further understanding the complex mechanisms of carotenoid synthesis in horticultural plants.

\section{Acknowledgements \\ This work was supported by the National Natural Science Foundation of China (31972362, 32001993), the Project of the Shandong Natural Science Foundation (ZR2020QC143), and the Breeding Plan of the Shandong Provincial Qingchuang Research Team (2019).}

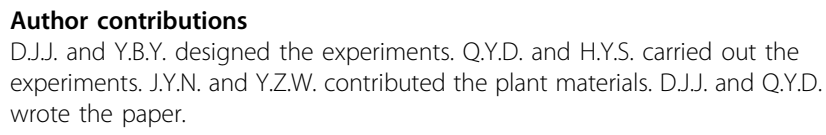

Supplementary information The online version contains supplementary material available at https://doi.org/10.1038/s41438-021-00694-w. 
Received: 20 April 2021 Revised: 15 August 2021 Accepted: 25 August 2021 Published online: 05 October 2021

\section{References}

1. Tanaka, Y., Sasaki, N. \& Ohmiya, A. Biosynthesis of plant pigments: anthocyanins, betalains, and carotenoids. Plant J. 54, 733-749 (2008).

2. Yuan, H., Zhang, J. X., Nageswaran, D. \& Li, L. Carotenoid metabolism and regulation in horticultural crops. Hortic. Res. 2, 15036 (2015).

3. Domonkos, I., Kis, M., Gombos, Z., \& Ughy, B. Carotenoids, versatile components of oxygenic photosynthesis. Prog. Lipid Res. 52, 539-561 (2013).

4. Fraser, P. D. \& Bramley, P. M. The biosynthesis and nutritional uses of carotenoids. Prog. Lipid Res. 43, 228-265 (2004).

5. Fiedor, J. \& Burda, K. Potential role of carotenoids as antioxidants in human health and disease. Nutrients 6, 466-488 (2014).

6. Cazzonelli, C. I. \& Pogson, B. J. Source to sink: regulation of carotenoid biosynthesis in plants. Trends Plant Sci. 15, 266-274 (2010).

7. Hao, Z., Liu, S., Hu, L., Shi, J. \& Chen, J. Transcriptome analysis and metabolic profiling reveal the key role of carotenoids in the petal coloration of Liriodendron tulipifera. Hor. Res. 7, 70 (2020).

8. Fraser, P. D. et al. Manipulation of phytoene levels in tomato fruit: effects on isoprenoids, plastids, and intermediary metabolism. Plant Cell 19, 3194-3211 (2007).

9. Rodriguez-Villalon, A., Gas, E. \& Rodriguez-Concepcion, M. Phytoene synthase activity controls the biosynthesis of carotenoids and the supply of their metabolic precursors in dark-grown Arabidopsis seedlings. Plant J. 60, 424-435 (2009).

10. Fraser, P. D. et al. Evaluation of transgenic tomato plants expressing an additional phytoene synthase in a fruit-specifc manner. Proc. Natl Acad. Sci. USA 99, 1092-1097 (2002).

11. Ducreux, L. J. et al. Metabolic engineering of high carotenoid potato tubers containing enhanced levels of beta-carotene and lutein. J. Exp. Bot. 56, 81-89 (2005).

12. Li, F., Vallabhaneni, R., Yu, J., Rocheford, T. \& Wurtzel, E. T. The maize phytoene synthase gene family: overlapping roles for carotenogenesis in endosperm, photomorphogenesis, and thermal stress tolerance. Plant Physiol. 147, 1334-1346 (2008).

13. Welsch, R., Wust, F., Bar, C., Al-Babili, S. \& Beyer, P. A third phytoene synthase is devoted to abiotic stress-induced abscisic acid formation in rice and defines functional diversification of phytoene synthase genes. Plant Physiol. 147, 367-380 (2008).

14. $\mathrm{Fu}, \mathrm{X}$. et al. Involvement of multiple phytoene synthase genes in tissue- and cultivar-specific accumulation of carotenoids in loquat. J. Exp. Bot. 65, 4679-4689 (2014).

15. Ampomah-Dwamena, C. The phytoene synthase gene family of apple (Malus $\times$ domestica) and its role in controlling fruit carotenoid content. BMC Plant Biol. 15, 185 (2015).

16. Jang, S. J. et al. Phytoene synthase 2 can compensate for the absence of PSY 1 in the control of color in Capsicum fruit. J. Exp. Bot. 71, 3417-3427 (2020).

17. Zhou, X. et al. Arabidopsis OR proteins are the major posttranscriptional regulators of phytoene synthase in controlling carotenoid biosynthesis. Proc. Natl Acad. Sci. USA 112, 3558-3563 (2015).

18. Park, S. et al. Orange protein has a role in phytoene synthase stabilization in sweet potato. Sci. Rep. 6, 33563 (2016).

19. Diretto, G. et al. Metabolic engineering of potato tuber carotenoids through tuber-specifc silencing of lycopene epsilon cyclase. BMC Plant Biol. 6, 13 (2006).

20. Jiang, F. et al. The apricot (Prunus armeniaca L.) genome elucidates Rosaceae evolution and beta-carotenoid synthesis. Hor. Res. 6, 128 (2019).

21. Sun, B. et al. Color-related chlorophyll and carotenoid concentrations of Chinese kale can be altered through CRISPR/Cas9 targeted editing of the carotenoid isomerase gene BoaCRTISO. Hor. Res. 7, 161 (2020).

22. Chayut, N. et al. Comparative transcriptome analyses shed light on carotenoid production and plastid development in melon fruit. Hor. Res. 8, 112 (2021).

23. Vrebalov, J. et al. Fleshy fruit expansion and ripening are regulated by the tomato SHATTERPROOF Gene TAGL1. Plant Cell. 21, 3041-3062 (2009).

24. Lu, S. et al. The citrus transcription factor CsMADS6 modulates carotenoid metabolism by directly regulating carotenogenic Genes. Plant Physiol. 176, 2657-2676 (2018)

25. Ma, N. N. et al. Overexpression of tomato SINAC1 transcription factor alters fruit pigmentation and softening. BMC Plant Biol. 14, 351 (2014).
26. Zhu, M. K. et al. A new tomato NAC (NAM/ATAF1/2/CUC2) transcription factor, SINAC4, functions as a positive regulator of fruit ripening and carotenoid accumulation. Plant Cell Physiol. 55, 119-135 (2014).

27. $\mathrm{Fu}, \mathrm{C}$. et al. The papaya transcription factor CpNAC1 modulates carotenoid biosynthesis through activating phytoene desaturase genes CPPDS2/4 during Fruit Ripening. J. Agric. Food Chem. 64, 5454-5463 (2016).

28. Sagawa, J. M. et al. An R2R3-MYB transcription factor regulates carotenoid pigmentation in Mimulus lewisii flowers. N. Phytol. 209, 1049-1057 (2016).

29. Zhu, F. et al. An R2R3-MYB transcription factor represses the transformation of a-and $\beta$-branch carotenoids by negatively regulating expression of $\mathrm{CrBCH} 2$ and CrNCED5 in flavedo of Citrus reticulate. N. Phytol. 216, 178-192 (2017).

30. Ampomah-Dwamena, C. et al. A kiwifruit (Actinidia deliciosa) R2R3-MYB transcription factor modulates chlorophyll and carotenoid accumulation. N. Phytol. 221, 309-325 (2019).

31. Meng, Y. et al. The MYB activator WHITE PETAL1 associates with MtTT8 and MtWD40-1 to regulate carotenoid-derived flower pigmentation in Medicago truncatula. Plant Cell. 31, 2751-2767 (2019).

32. Zhou, D. et al. Papaya CpbHLH1/2 regulate carotenoid biosynthesis-related genes during papaya fruit ripening. Hor. Res. 6, 80 (2019).

33. Yang, X. et al. The histone variant SI_H2A.Z regulates carotenoid biosynthesis and gene expression during tomato fruit ripening. Hor. Res. 8, 85 (2021).

34. Girardi, C. L. et al. Genome-wide analysis of the AP2/ERF superfamily in apple and transcriptional evidence of ERF involvement in scab pathogenesis. Sci. Horticulturae 151, 112-121 (2013).

35. Cheng, L. et al. Identifcation of AP2/ERF genes in apple (Malus $\times$ domestica) and demonstration that MdERF017 enhances iron deficiency tolerance. Plant Cell Tiss. Org. 143, 465-482 (2020).

36. Shi, W. et al. Liu X. StRAP2.3, an ERF-VII transcription factor, directly activates StInvinh2 to enhance cold-induced sweetening resistance in potato. Hor. Res. 8, 82 (2021).

37. Zhao C. et al. Three AP2/ERF family members modulate flavonoid synthesis by regulating type IV chalcone isomerase in citrus. Plant Biotechnol. J. https://doi. org/10.1111/pbi.13494 (2020).

38. Wang, $X$. et al. PpERF3 positively regulates $A B A$ biosynthesis by activating PpNCED2/3 transcription during fruit ripening in peach. Hor. Res. 6, 19 (2019).

39. Lee, J. M. et al. Combined transcriptome, genetic diversity and metabolite profiling in tomato fruit reveals that the ethylene response factor SIERF6 plays an important role in ripening and carotenoid accumulation. Plant J. 70, 191-204 (2012).

40. Welsch, R., Maass, D., Voegel, T., DellaPenna, D. \& Beyer, P. Transcription factor RAP2.2 and its interacting partner SINAT2: stable elements in the carotenogenesis of Arabidopsis leaves. Plant Physiol. 145, 1073-1085 (2007).

41. Ampomah-Dwamena, C. et al. Metabolic and gene expression analysis of apple (Malus $\times$ domestica) carotenogenesis. J. Exp. Bot. 63, 4497-511 (2012).

42. Jia, D. J. et al. Apple fruit acidity is genetically diversified by natural variations in three hierarchical epistatic genes MdSAUR37, MdPP2CH and MdALMTII. Plant J. 95, 427-443 (2018).

43. Jia, D. J. et al. Genetic transformation of the astaxanthin biosynthetic genes bkt and $c r t R-B$ into apple tree to increase photooxidation resistance. Sci. Horticulturae. 243, 428-433 (2019).

44. Jia, D. J. et al. Anthocyanin biosynthesis and methylation of the MdMYB10 promoter are associated with the red blushed-skin mutant in the red stripedskin 'Changfu 2' apple. J. Agr. food chem. 68, 4292-4304 (2020).

45. Jia, D. J. et al. Genetic variation in the promoter of an R2R3-MYB transcription factor determines fruit malate content in apple (Malus domestica Borkh.). Plant Physiol. 186, 549-568 (2021).

46. Da, L. et al. AppleMDO: a multi-dimensional omics database for apple coexpression networks and chromatin states. Plant Sci. Front. 10, 1333 (2019).

47. Nole-Wilson, S., Tranby, T. L. \& Krizek, B. A. AINTEGUMENTA-like (AIL) genes are expressed in young tissues and may specify meristematic or divisioncompetent states. Plant Mol. Biol. 57, 613-628 (2005).

48. Liu, Q. et al. Two transcription factors, DREB1 and DREB2, with an EREBP/AP2 DNA binding domain separate two cellular signal transduction pathways in drought-and low-temperature-responsive gene expression, respectively, in Arabidopsis. Plant Cell 10, 1391-1406 (1998).

49. Sakuma, Y. et al. DNA-binding specificity of the ERF/AP2 domain of Arabidopsis DREBs, transcription factors involved in dehydration- and cold- inducible gene expression. Biochem. Biophys. Res. Comm. 290, 998-1009 (2002).

50. Wang, M. M. et al. Hypoxia-responsive ERFs involved in postdeastringency softening of persimmon fruit. Plant Biotechnol. J. 15, 1409-1419 (2017). 